\title{
Study on New Non Gas Source Negative Pressure Adsorption Structure Key Laboratory for Control Theory \& Applications in Complicated Systems
}

\author{
Lei Shao ${ }^{\mathrm{a}}$, Hongyu Li ${ }^{\mathrm{b}}$, Hongli Liu' ${ }^{\mathrm{c}}$, Ji Lic, Xiaoqi Chen ${ }^{\mathrm{c}}$ \\ School of Electrical and Electronic Engineering, Tianjin University of Technology, Tianjin 300384, \\ China \\ a1794482484@qq.com, b1794482484@qq.com, c1794482484@qq.com
}

Keywords: Passive, Negative pressure suction, Sucker, power consumption, load capacity.

\begin{abstract}
In order to overcome the disadvantages of the existing negative pressure adsorption methods, a new structure of non gas source negative pressure adsorption is proposed. On the basis of this structure, a physical prototype was made. According to the motion model of the prototype, the stability condition of the wall adsorption of the prototype was analyzed.The load capacity test of the prototype was carried out to find out the relationship between the load capacity and the material, diameter and thickness of the sucker. The experiment of the prototype shows that the design of this structure is reasonable, and the adsorption force is strong and stable.
\end{abstract}

\section{Introduction}

Wall climbing robot is an important branch in the field of mobile robot, it combine the ground mobile robot technology and adsorption technology organically, it can be attached to the vertical wall crawling, and can carry tools to complete certain tasks, greatly expanded the application scope of robot. The wall climbing robot has three adsorption manners: negative pressure adsorption, magnetic adsorption and other adsorption methods. Among them, negative pressure adsorption methods usually use vacuum pumps to provide negative pressure. The method has the disadvantages of high power consumption, large noise and complicated structure. It cannot meet the requirements of practical work. In order to overcome the above defects, reference [1] designed a kind of biomimetic adsorption mechanism imitating tapeworm adsorption mechanism, including IPM (ion-exchange polymer metal composite) driver chuck and small hook structure; reference [2] proposed Octopus suckers model, based on analyzing the structure characteristics of biological organs by vacuum adsorption, bias type shape shape memory alloy (shape memory alloy, SMA) spring actuator design a bionic sucker. Nowadays, the research trend of negative pressure adsorption method is developing towards bionics. As mentioned above, the absorption mechanism for mimicking the tapeworm mechanism and the octopus sucker is developed by imitating the movements of animals and humans such. The principle and structure of this method are complex and difficult to control. In this paper, a new non gas source negative pressure adsorption structure is designed by imitating the principle of negative pressure produced by simple sucker hanger. And a physical prototype is constructed on the basis of this structure. The stability condition of the prototype is analyzed, and the load capacity test of the prototype is done to verify the analysis results.

\section{Passive adsorption structure}

In order to solve the defects of the existing negative pressure adsorption method, a new Passive negative pressure adsorption structure with simple structure and reliable adsorption is designed by simulating the negative pressure producing principle of a simple sucker hanger (Fig. 1).

As shown in Figure 2, pressure bar 10, spring 8 and sucker 5 constitute the negative adsorption structure. The adsorption structure is arranged on a chassis and cooperates with the chassis to complete the adsorption action. The end of the pressure bar 10 is connected to the sucker 5 , and the 
sucker 5 can be pressed and pulled by the pressure bar 10.The upper end of the spring 8 is connected to the pressure bar 10 and its lower end is connected to the chassis. Spring 8 can be contracted and elongated with the action of the pressure bar 10.

\subsection{Vacuum adsorption processes.}

Pressure bar 10 presses the sucker 5, so that air between the sucker 5 and the climbing surface is ejected, the spring 8 is also is squeezed to produce deformation. When the gas is exhausted, the pressure bar 10 removes the pressure of the sucker 5 and the spring 8 , then the spring 8 restores its original state and the elasticity of spring drives the pressure bar 10 upward to pull the sucker 5, so that the vacuum environment is formed between the sucker 5 and the wall surface.

\subsection{Vacuum adsorption release processes.}

Pressure bar 10 pulls sucker 5 so that the sucker 5 is separated from the wall surface. After the sucker 5 is disengaged, the pulling force of the sucker and the spring is removed, then the adsorption device is restored to the initial state.

The pressing and pulling of the pressure bar 10 is done by the driver on the chassis. As shown in Figure 2, driver is made up of sucker motor 7 and cam 6 . The sucker motor 7 drives the cam 6 forward, and the cam drives the pressure bar to press the sucker. The sucker motor 7 drives the cam backward to pull the sucker.

Once the vacuum environment is formed, the driver can stop working. The negative pressure is only maintained by the elasticity of spring. Its power consumption is almost zero when the adsorption device is quietly adsorbed on the surface of wall.

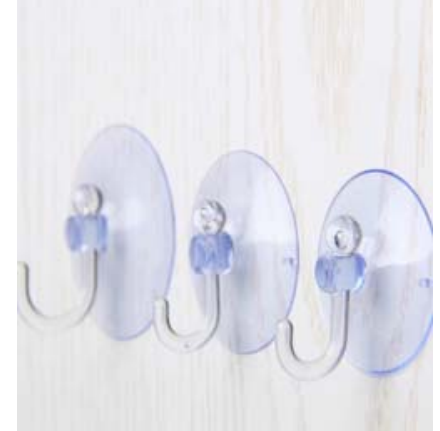

Fig.1.Simple sucker hanger

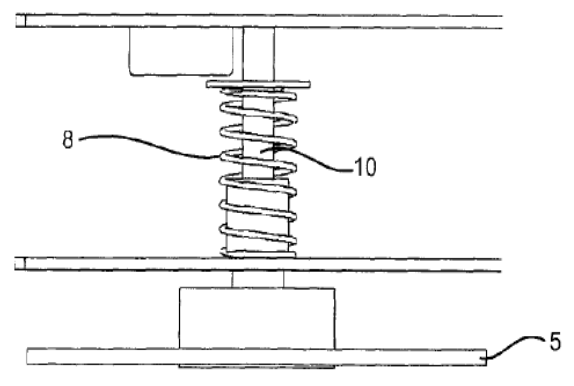

Fig.2.Passive adsorption structure

\section{Mechanical structure design of prototype}

On the basis of the above passive adsorption structure, we select the track as the moving structure and produce a physical prototype of Fig.2

As shown in Fig.3, the prototype consists of seven parts, namely, chassis 1, track 2, track motor 3, adsorption structure 4, cam 6, sucker motor 7, and controller 9 .

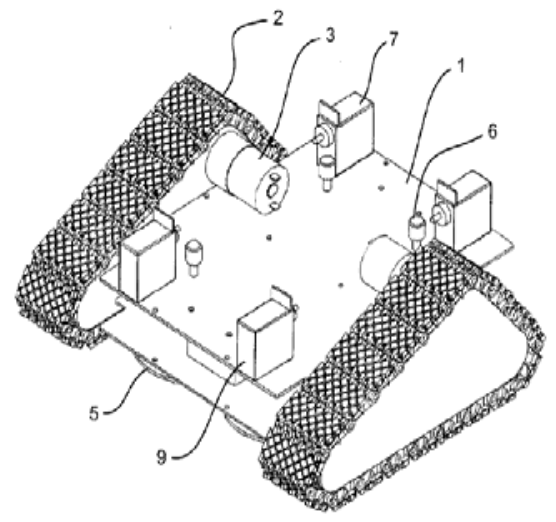

Fig.3.Mechanical structure of prototype

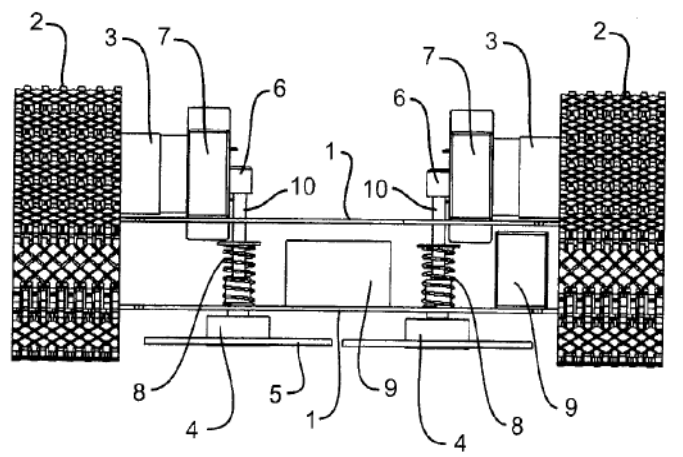

Fig.4.Mechanical structure of prototype

Among them, the moving structure of the robot consists of track 2 and track motor 3, there is a group both on the left and the right. There are four groups of adsorption structure 4 which is installed 
on the chassis 1 . The cam 6 and the sucker motor 7 assists the adsorption mechanism 4 to complete the adsorption action.

When the adsorption structure start sucker, the robot adsorbed on the surface of the wall, track motor 3 drive track 2 to rotate forward, so that the robot moving forward. The two sides of the track motor 3 change their direction to make the robot forward, backward or turn.

During the robot's travel, at least one sucker 3 remains stuck and slides along the surface of the climbing wall, the robot need not only to overcome its own gravity, but also to overcome the sliding friction between the suction drum and the wall, therefore, the outer surface of the sucker needs a low enough friction coefficient to allow the sucker to slide on the wall and maintain the adsorption-force at the same time.

Both adsorption action of the adsorption structure and the action of the moving structure are under the control of controller 9 .

A pressure sensor can also be installed on each adsorption structure. The sensor can measure the adsorption-force of the sucker and pass it to the controller, so the controller can judge whether the suction cup is leaking or not, and take measures to restart the sucker in time.

\section{Steady conditions for uniform driving}

Whether the wall-climbing robot can be safely and stably adsorbed on the vertical wall depends on its force and moment balance. When the robot crawls in the vertical plane, it must be balanced under the influence of the wall friction and gravity. Otherwise, there will be two possible forms of failure. One is sliding along the wall, and another is the sucker out of the wall, causing the robot to turn over.

\subsection{Force balance analyses.}

As shown in Figure 5, only if meet formula (1), the prototype does not glide when traveling at the vertical surface at uniform speed.

In formula (1), F2 is the static friction of the track, f1 is the sliding friction of the suction cup, G1 is the gravity of the prototype itself, and the G2 is the Loading of prototype.

$$
F_{2}=4 \mathrm{f}_{1}+G_{1}+G_{2}
$$

Suppose that the negative pressure $\Delta \mathrm{P}$ of the four suckers of the prototype is the same, the adsorption area $\mathrm{S}$ is the same, so that the negative pressure $\mathrm{f}$ of the sucker is the same. Driving force $F_{1}$ of motor to track is equal to the static friction. $F_{2}$ of the track. Sliding friction coefficient of sucker is $\mathrm{u}$.

$\mathrm{f}_{1}=\mathrm{fu}=\Delta P S \mathrm{u}$

$G_{2}=F_{1}-G_{1}-4 \triangle P S \mathrm{u}$

When the prototype is about to decline, the driving power of the motor reaches its maximum value $\mathrm{F}$,the Loading of prototype reaches the maximum value $\mathrm{G}_{3}$

$$
G_{3}=F-G_{1}-4 \Delta P S u
$$

In formula (4), $F$ and $\mathrm{G}_{1}$ are immutable, the maximum load value $\mathrm{G}_{3}$ of prototype is determined by the negative pressure $\Delta \mathrm{P}$, the adsorption area $\mathrm{S}$ and the sliding friction coefficient $\mathrm{u}$ of the sucker. However, the size of these three variables is determined by the material and diameter of the sucker. So the maximum load value of prototype is determined by the material and diameter of the sucker, when traveling at the vertical surface at uniform speed.

\subsection{Moment balance analyses.}

When the prototype is attached to the vertical wall, the force in the vertical direction is the gravity $\mathrm{G}$ of prototype. The force in a horizontal direction is negative pressure $\mathrm{T}$ of the sucker and the supporting force of the wall to the track. The robot will not capsize until torque is balanced in the B point.

The B point is the lowest end of the contact between the prototype and the wall, the A point is the upper end of the contact between the prototype and the wall, the $\mathrm{O}$ point is the center of gravity of robot, the $\mathrm{O}_{1}, \mathrm{O}_{2}$ point is the center point of the sucker. The distance between $\mathrm{A}$ and $\mathrm{B}$ is $\mathrm{l}$, The distance between $\mathrm{O}_{1}$ and $\mathrm{O}_{2}$ is $\mathrm{l}_{1}$ and $\mathrm{l}_{2}$, The distance between the $\mathrm{O}$ point and the wall is d. The 
equivalent resultant force of support force of the wall to the track is N.As shown in Figure 6,only if meet formula (5),the prototype does not overturn when traveling at the vertical surface at uniform speed.

$$
\begin{aligned}
& T l_{1}+T l_{2}=G d+2 N \bullet 1 / 2 l \\
& N=\frac{T l_{1}+T l_{2}-G d}{l}
\end{aligned}
$$

Only if $\mathrm{N}>0$, prototype does not overturn, so we can get the formula (7).

$$
T>\frac{G d}{l_{1}+l_{2}}
$$

After the size and weight of the robot body are determined, the negative pressure of the sucker must meet the formula (7).

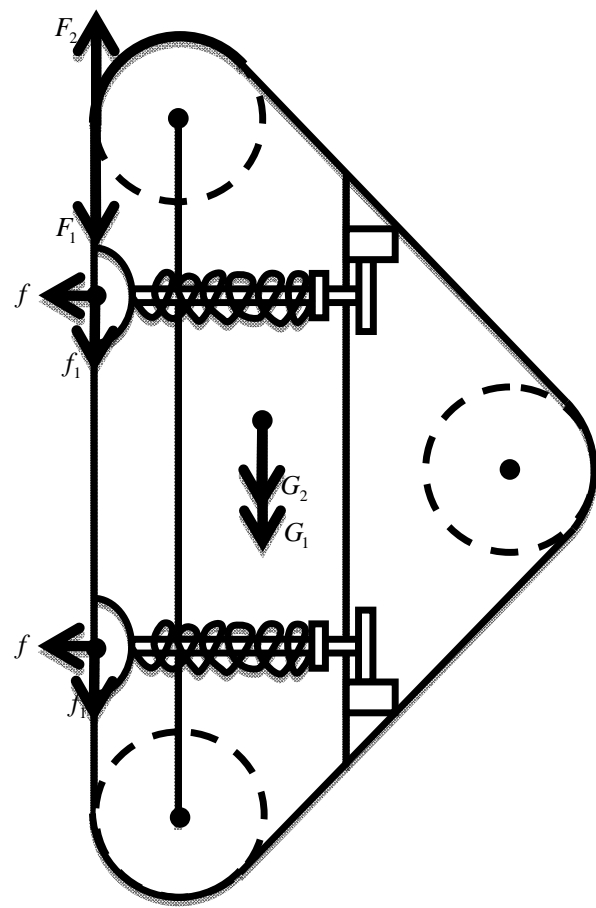

Fig.5.Force balance model

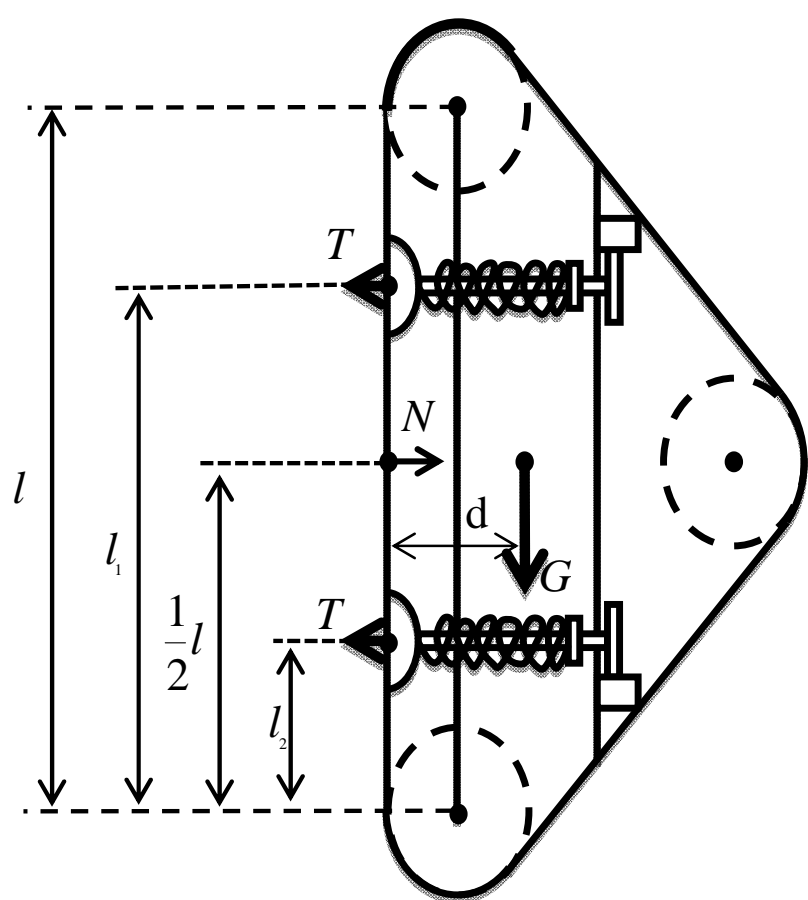

Fig.6.Moment balance model

\section{Prototype experiment}

The physical prototype we have made is shown in figure 7. In order to further study of the stability condition of uniform driving, we select a series of suckers with different parameters to test the load capacity of the prototype.

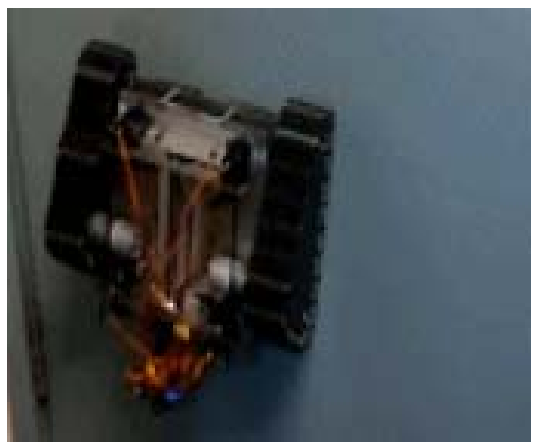

Fig.7.Prototype

We establish the mechanical model to analyze the stability condition of the prototype at uniform speed. The following conclusions are drawn: the maximum load capacity of the prototype is directly related to the material and diameter of the sucker. In order to verify the above conclusions, we choose "nitrile", "natural rubber" and "embedded reinforcement reinforced rubber" three kinds of test 
materials, and manufacture several groups of different thickness, diameter of suction, and install them on a prototype in turn, to complete weight capacity experiment. As shown in Table 1, the test is tested five times for each change in the sucker parameter during the test. We have plotted the curves as shown in Table 7 according to table 1.

The experimental data show that if the sucker is made of different materials under the same diameter and thickness of the sucker, the load capacity of the suction cup will be also different.

The best sucker material is reinforced rubber. It has the largest load capacity under the condition of providing a reliable sealed vacuum environment and a low enough sliding friction coefficient when the prototype is climbing on the surface of the wall.

The load capacity of the prototype increases as the diameter of the suction cup increases, and the general trend is shown in the curve of figure 7 . When the diameter of the suction cup is above $100 \mathrm{~mm}$, the load capacity of individual samples is negative correlation to the diameter of the sucker. Through observation and analysis, we can see that the trend is caused by irregular deformation of sucker.

For most materials, the $100 \mathrm{~mm}$ diameter is the best choice and can provide the maximum load capacity for the prototype.

Table.1.Experimental data of weight-bearing capacity

\begin{tabular}{|c|c|c|c|c|c|c|c|c|c|c|c|c|c|c|c|c|c|}
\hline \multirow{2}{*}{ Material type } & \multirow{2}{*}{$\begin{array}{c}\text { Material } \\
\text { Thickness } \\
\text { [mm] }\end{array}$} & \multirow[b]{2}{*}{$\begin{array}{c}\text { Pad } \\
{[\mathrm{mm}]}\end{array}$} & \multicolumn{8}{|c|}{ Run } & \multicolumn{7}{|c|}{ Analysis } \\
\hline & & & & $\mathrm{BL}$ & ${ }^{2}$ & & $\begin{array}{c}3 \\
M L\end{array}$ & $\mathrm{BL}$ & $\begin{array}{r}{ }^{4} \\
M L\end{array}$ & BL & & & $\begin{array}{l}\text { Av- } \\
\text { erage }\end{array}$ & MinML & MaxBL & $\begin{array}{l}\text { Error } \\
\text { (+ve) }\end{array}$ & $\begin{array}{l}\text { Error } \\
\text { (-ve) }\end{array}$ \\
\hline \multirow[t]{5}{*}{ Natural Rubber } & 3.8 & 80 & 24 & 26 & 24 & 26 & 24 & 26 & 24 & 24 & 24 & 26 & 25.48 & 24.68 & 26.68 & 1.2 & 0.8 \\
\hline & & 90 & 28 & 30 & 27 & 29 & 27 & 29 & 27 & 29 & 27 & 29 & 28.88 & 27.68 & 30.68 & 1.8 & 1.2 \\
\hline & & 100 & 31 & 31 & 30 & 30 & 30 & 30 & 29 & 31 & 29 & 29 & 30.68 & 29.68 & 30.68 & 1 & 1 \\
\hline & & 110 & 29 & 31 & 28 & 28 & 32 & 32 & 30 & 32 & 30 & 30 & 30.88 & 28.68 & 32.68 & 1.8 & 2.2 \\
\hline & & 120 & 20 & 22 & 20 & 22 & 20 & 22 & 20 & 22 & 20 & 22 & 21.68 & 30.68 & 22.68 & 1 & 1 \\
\hline \multirow[t]{4}{*}{ Natural Rubber } & 4.8 & 80 & 27 & 29 & 27 & 29 & 28 & 28 & 28 & 28 & 27 & 29 & 28.68 & 27.68 & 29.68 & 1 & 1 \\
\hline & & 90 & 32 & 32 & 32 & 32 & 32 & 32 & 30 & 32 & 32 & 32 & 32.48 & 30.68 & 32.68 & 0.2 & 1.8 \\
\hline & & 110 & 32 & 34 & 34 & 34 & 32 & 34 & 34 & 34 & 30 & 30 & 33.48 & 30.68 & 34.68 & 1.2 & 2.8 \\
\hline & & 120 & 41 & 43 & 41 & 43 & 39 & 41 & 39 & 41 & 39 & 41 & 41.48 & 39.68 & 43.68 & 2.2 & 1.8 \\
\hline \multirow[t]{5}{*}{ Nitrile } & 3 & 80 & 26 & 28 & 27 & 29 & 27 & 27 & 27 & 29 & 27 & 29 & 28.28 & 26.68 & 29.68 & 1.4 & 1.6 \\
\hline & & 90 & 31 & 33 & 30 & 32 & 30 & 32 & 31 & 33 & 32 & 32 & 32.28 & 30.68 & 33.68 & 1.4 & 1.6 \\
\hline & & 100 & 34 & 34 & 32 & 32 & 30 & 30 & 30 & 32 & 30 & 32 & 32.28 & 30.68 & 34.68 & 2.4 & 1.6 \\
\hline & & 110 & 32 & 34 & 25 & 25 & 20 & 20 & 30 & 30 & 20 & 20 & 20.68 & 19.68 & 34.68 & 8.4 & 5.6 \\
\hline & & 120 & 20 & 22 & 20 & 20 & 20 & 20 & 20 & 20 & 19 & 19 & 20.68 & 19.68 & 22.68 & 2 & 1 \\
\hline \multirow[t]{5}{*}{ Insertion reinforced } & 3 & 80 & 32 & 34 & 32 & 34 & 32 & 34 & 32 & 32 & 32 & 34 & 33.68 & 32.68 & 34.68 & 1 & 1 \\
\hline & & 90 & 39 & 41 & 41 & 39 & 39 & 39 & 39 & 39 & 39 & 39 & 40.08 & 39.68 & 41.68 & 1.6 & 0.4 \\
\hline & & 100 & 43 & 45 & 43 & 45 & 43 & 43 & 45 & 43 & 43 & 43 & 44.28 & 43.68 & 45.68 & 1.4 & 0.6 \\
\hline & & 110 & 43 & 43 & 41 & 41 & 41 & 43 & 41 & 41 & 39 & 39 & 41.88 & 39.68 & 43.68 & 1.8 & 2.2 \\
\hline & & 120 & 35 & 35 & 35 & 35 & 35 & 35 & 35 & 35 & 35 & 35 & 35.68 & 35.68 & 35.68 & 0 & 0 \\
\hline \multirow[t]{3}{*}{ Insertion reinforced 4.8} & 4.8 & 80 & 32 & 32 & 32 & 34 & 32 & 34 & 32 & 32 & 32 & 32 & 33.08 & 32.68 & 34.68 & 1.6 & 0.4 \\
\hline & & 90 & 41 & 43 & 41 & 41 & 43 & 41 & 41 & 41 & 41 & 43 & 42.48 & 41.68 & 43.68 & 1.2 & 0.8 \\
\hline & & 100 & 51 & 51 & 51 & 53 & 51 & 53 & 51 & 53 & 51 & 53 & 52.48 & 51.68 & 53.68 & 1.2 & 0.8 \\
\hline \multirow[t]{7}{*}{ Insertion 2x reinforced } & 7.5 & 110 & 53 & & 53 & & 53 & & 53 & & 53 & & 53.68 & 53.68 & 53.68 & 0 & 0 \\
\hline & & 120 & 53 & & 53 & & 53 & & 53 & & 53 & & 53.68 & 53.68 & 53.68 & 0 & 0 \\
\hline & & 80 & 30 & 35 & 32 & 32 & 34 & 36 & 34 & 34 & 34 & 34 & 34.18 & 30.68 & 36.68 & 2.5 & 2.5 \\
\hline & & 90 & 41 & 43 & 41 & 43 & 43 & 43 & 43 & 43 & 43 & 43 & 43.28 & 41.68 & 43.68 & 0.4 & 1.6 \\
\hline & & 100 & 49 & 51 & 53 & 53 & 53 & 53 & 53 & 53 & 53 & 53 & 53.08 & 49.68 & 53.68 & 0.6 & 3.4 \\
\hline & & 110 & 53 & & 53 & & 53 & & 53 & & 53 & & 53.68 & 53.68 & 53.68 & 0 & 0 \\
\hline & & 120 & 53 & & 53 & & 53 & & 53 & & 53 & & 53.68 & 53.68 & 53.68 & 0 & 0 \\
\hline
\end{tabular}




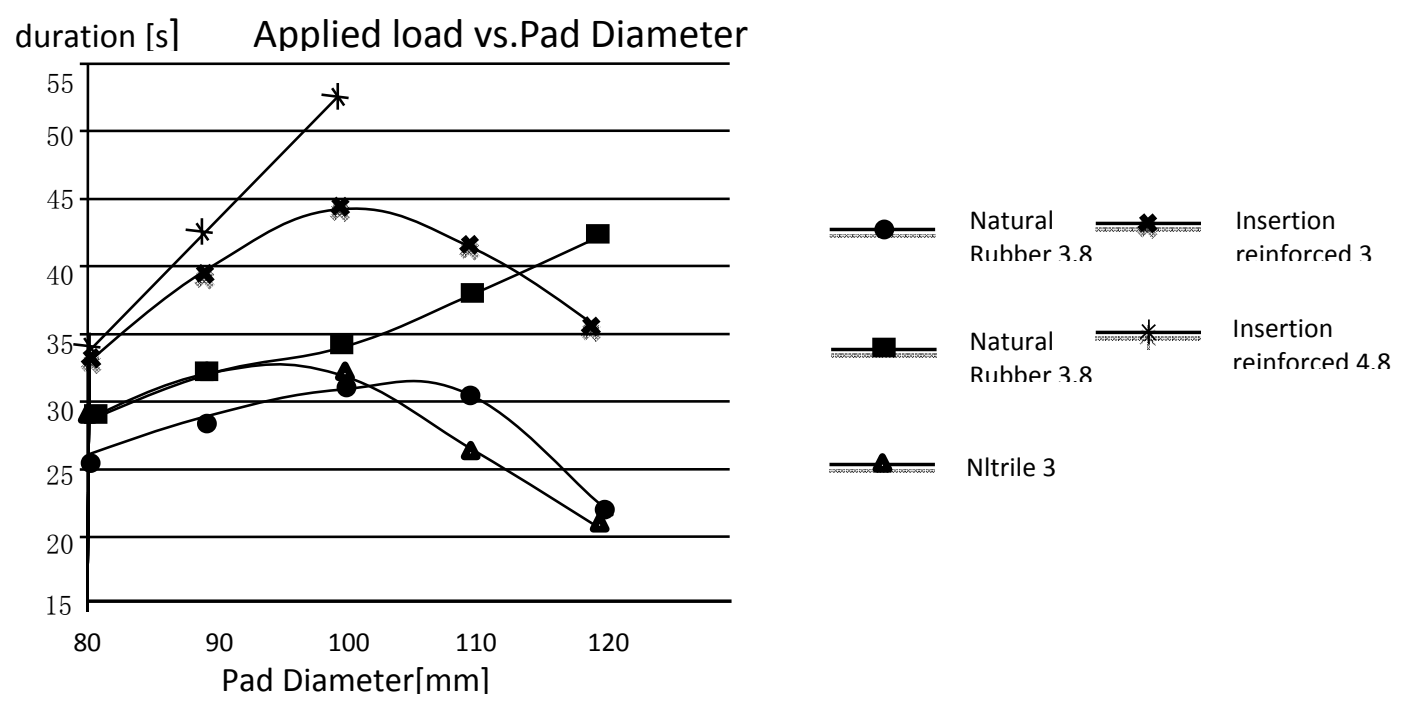

Fig.8.Load capacity curve

The above experimental data verify the conclusion that the load capacity of the prototype is directly related to the material and diameter of the sucker when running at uniform speed.

During the experiment, we also found that the increase of the thickness of the sucker can also improve the load capacity of the prototype. Table 1 shows that the thickness of the sucker is at least $3.8 \mathrm{~mm}$, the maximum is $7.5 \mathrm{~mm}$, and the optimum thickness is $4.8 \mathrm{~mm}$.

In summary, the highest quality sucker for non gas source negative pressure adsorption structure proposed in this paper is an enhanced rubber sucker with diameter $100 \mathrm{~mm}$ and thickness $4.8 \mathrm{~mm}$.

\section{Summary}

In this paper, a novel non gas source negative pressure adsorption structure with simple structure and reliable adsorption is designed by imitating the principle of negative pressure produced by sucker plate hook. On the basis of this structure, a physical prototype is built, and a mechanical model of the prototype is established to analyze the equilibrium condition of the prototype. The load capacity test of the prototype shows that the passive adsorption mechanism is reasonable, and the adsorption force is strong and stable. At the same time confirmed the highest quality sucker for passive adsorption structure proposed in this paper is an enhanced rubber sucker with diameter $100 \mathrm{~mm}$ and thickness $4.8 \mathrm{~mm}$.

\section{Acknowledgements}

This work was financially supported by the Tianjin science and technology plan project (15ZXZNGX00140) and Tianjin applied basic research project-Tianjin science and technology special commissioner project (16JCTPJC49400).

\section{References}

[1] MENCIASSIA, DARIOP. Bio-inspired solutions for locomotion in the gastrointestinal tract: background and perspectives. Philosophical Transactions of the Royal Society A: Mathematical, Physical and Engineering Sciences.(2003), p: 2287-2298

[2] GRASSOWF, SETLURP. Inspiration, simulation and design for smart robot manipulators from the sucker actuation mechanism of cephalopods. Bioinspiration and Biomimetics. (2007)No.4, p: 170-181

[3] Yili Fu, Zhihai Li. Research progress of wall climbing robot. Mechanical design,.Vol.25(2008). No4, p: $1-5$ 
[4] Mingqin Liu, Yongxiong Dai, Wenpan Huang, Maofei Fan, Bin Qiao. Mechanical structure and balance of a small climbing wall climbing robot. Mechanical design \& manufacture,. (2011).No5, p: 146-148

[5] Zhu Zhihong, Li Jize, Peng Jinmin, Li Jun, high Xueshan. Micro wall climbing robot mobile detection platform research. Journal of mechanical engineering Vol.47.(2011)No.4, p: 49-54

[6] Li Zhihai, Fu Yi Li, Wang Shuguo. Study on the dynamics of four wheel drive sliding suction wall climbing robot. The robot.Vol.32. (2010)No5, p: 601-607 